\title{
Production of Sulfur Allotropes in Electron Irradiated Jupiter Trojans Ice Analogs
}

\author{
Ahmed Mahjoub $^{1}$ (1) , Michael J. Poston ${ }^{1,2}$, Jordana Blacksberg ${ }^{1}$, John M. Eiler ${ }^{2}$, Michael E. Brown ${ }^{2}$ (1) , Bethany L. Ehlmann ${ }^{1,2}$ (1), \\ Robert Hodyss $^{1}$, Kevin P. Hand ${ }^{1}$ (D), Robert Carlson ${ }^{1}$, and Mathieu Choukroun ${ }^{1}$ \\ ${ }^{1}$ Jet Propulsion Laboratory, California Institute of Technology, Pasadena, CA 91109, USA; Mahjoub.Ahmed@jpl.nasa.gov \\ ${ }^{2}$ California Institute of Technology, Division of Geological and Planetary Sciences, Pasadena, CA 91125, USA \\ Received 2017 May 13; revised 2017 August 1; accepted 2017 August 8; published 2017 September 12
}

\begin{abstract}
In this paper, we investigate sulfur chemistry in laboratory analogs of Jupiter Trojans and Kuiper Belt Objects (KBOs). Electron irradiation experiments of $\mathrm{CH}_{3} \mathrm{OH}-\mathrm{NH}_{3}-\mathrm{H}_{2} \mathrm{O}$ and $\mathrm{H}_{2} \mathrm{~S}-\mathrm{CH}_{3} \mathrm{OH}-\mathrm{NH}_{3}-\mathrm{H}_{2} \mathrm{O}$ ices were conducted to better understand the chemical differences between primordial planetesimals inside and outside the sublimation line of $\mathrm{H}_{2} \mathrm{~S}$. The main goal of this work is to test the chemical plausibility of the hypothesis correlating the color bimodality in Jupiter Trojans with sulfur chemistry in the incipient solar system. Temperature programmed desorption (TPD) of the irradiated mixtures allows the detection of small sulfur allotropes $\left(\mathrm{S}_{3}\right.$ and $\left.\mathrm{S}_{4}\right)$ after the irradiation of $\mathrm{H}_{2} \mathrm{~S}$ containing ice mixtures. These small, red polymers are metastable and could polymerize further under thermal processing and irradiation, producing larger sulfur polymers (mainly $\mathrm{S}_{8}$ ) that are spectroscopically neutral at wavelengths above $500 \mathrm{~nm}$. This transformation may affect the spectral reflectance of Jupiter Trojans in a different way compared to KBOs, thereby providing a useful framework for possibly differentiating and determining the formation and history of small bodies. Along with allotropes, we report the production of organosulfur molecules. Sulfur molecules produced in our experiment have been recently detected by Rosetta in the coma of 67P/Churyumov-Gerasimenko. The very weak absorption of sulfur polymers in the infrared range hampers their identification on Trojans and KBOs, but these allotropes strongly absorb light at UV and Visible wavelengths. This suggests that high signal-to-noise ratio UV-Vis spectra of these objects could provide new constraints on their presence.
\end{abstract}

Key words: astrochemistry - Kuiper belt: general - methods: laboratory: molecular - molecular processes techniques: imaging spectroscopy

\section{Introduction}

Small bodies are the legacy left from the building blocks that accreted to create planets in the early solar system. Studying small objects such as Jupiter Trojans and Kuiper Belt Objects (KBOs) provides a unique perspective on the chemical and dynamical processes that shaped the solar system and a particular opportunity to evaluate new ideas about the formation and dynamical evolution of the solar system. In the framework of the Nice model, KBOs and Trojans are thought to have formed in the primordial planetesimal disk in the nascent solar system, with progenitors sourced from the same regions of the disk (Morbidelli et al. 2005; Nesvorny et al. 2013). As a result of dynamical instability and migration of giant planets, objects among this primordial disk of debris were dispersed, giving rise to the Jupiter Trojans and KBOs in their current positions, as well as other small objects. One of the most tantalizing properties of these two populations is the color bimodality of visible/near-infrared spectral slopes observed in both of them. Two classes of Trojans are distinctly identified by telescopic observations and referred to as red (R) and less red (LR) (Emery et al. 2011). A dynamical sub-population within the KBO family ("hot" KBOs) shows two sub-populations referred to as very red (VR) and red (R) KBOs (Brown et al. 2011; Wong \& Brown 2016, 2017). This similarity in spectral slope bimodality is in accordance with a common origin for these two groups of objects. Moreover, the size distributions of Trojans and hot KBOs are consistent with each other, which supports the idea of a shared formation location (Fraser et al. 2014).
Surfaces of icy, airless bodies are exposed to photons and energetic particles from the Sun. This space weathering is believed to be responsible for the growth of organic-bearing refractory mantles on the surfaces of small icy bodies. There are increasing indications that organic polymers are present in primitive bodies in the solar system. For example, such refractory materials are considered to be a good spectral analog for the $3.2 \mu \mathrm{m}$ band observed at the surface of comet $67 \mathrm{P} /$ ChuryumovGerasimenko, hereafter 67P, by the VIRTS/Rosetta spectrometer (Quirico et al. 2016). These complex macromolecular organic materials are believed to play an important role in the red spectral slopes observed in small bodies in the solar system (Gradie \& Veverka 1980) and are included in spectral modeling of Trojans (Emery et al. 2011). In one hypothesis, the bimodality in colors could be a result of differing ice compositions between the two populations. The retention of volatiles on the surface of objects in the outer primordial planetesimal disk depends on their orbital distances. Using a volatile loss model, Wong \& Brown (2016) found that the stability line of $\mathrm{H}_{2} \mathrm{~S}$ passes through the region of the primordial disk, splitting objects into two groups: with sulfur and without sulfur. They used this location-dependent surface composition to propose a hypothesis explaining the surface color bimodality observed today for Trojans and KBOs as result of sulfur chemistry. This hypothesis is relevant only to small KBOs.

Small sulfur allotropes strongly absorb in the UV-Vis range, and the absorption wavelengths depend on the chain length (Meyer 1976). Meyer (1976) showed that the absorption of sulfur chains in UV-Vis range shifts toward high wavelengths with increasing numbers of $\mathrm{S}$ atoms, and reach an asymptote at $800 \mathrm{~nm}$ for chains containing five S atoms or more. Small 
allotropes exhibit high degrees of temperature and irradiation instability. A direct test of the thermal polymerization of small sulfur allotropes was performed by Brabson et al. (1991). In their study, Brabson et al. (1991) applied a microwave discharge in sulfur vapor and condensed the obtained gas at $12 \mathrm{~K}$. Using infrared spectroscopy coupled with isotopic shift analysis they convincingly concluded that the samples produced are mainly composed of $\mathrm{S}_{2}, \mathrm{~S}_{3}$, and $\mathrm{S}_{4}$, with less abundance of higher sulfur chains. Annealing these samples results in a decrease of allotrope bands and an increase in bands assigned to larger cyclic sulfur molecules.

It is well established that melting a sulfur powder at temperatures higher than $250^{\circ} \mathrm{C}$ modifies its color from yellow to red. By using UV-Vis spectroscopy, Meyer et al. (1971) attributed this change in color to the formation of allotropes. Cooling the liquid down to $70 \mathrm{~K}$, does not change the color of the sample, which is interpreted as a preservation of sulfur allotropes at this temperature. Warming the sample from $70 \mathrm{~K}$ to room temperature changes the color of the sample to its original color: yellow. This has been interpreted as a consequence of the polymerization of unstable small sulfur chains to orthorhombic $S_{8}$. Similar observations were made after irradiation and thermal processing of $\mathrm{S}_{2} \mathrm{O}$ ice samples (Baklouti et al. 2008). In Baklouti et al. (2008), the change of color from red to yellow is accompanied by the appearance of IR bands due to polysulfuroxide.

To gain insight into the role of $\mathrm{H}_{2} \mathrm{~S}$ on chemical and spectral properties of small primitive objects, we have conducted laboratory experiments testing the influence of sulfur chemistry on spectral slope in the visible-NIR range when it is added to a $\mathrm{C}-$, N-, and O-bearing ice mixture. Mixed ices $\mathrm{H}_{2} \mathrm{~S}-\mathrm{CH}_{3} \mathrm{OH}-\mathrm{NH}_{3}-\mathrm{H}_{2} \mathrm{O}$ "with $\mathrm{H}_{2} \mathrm{~S}$ " and $\mathrm{CH}_{3} \mathrm{OH}-\mathrm{NH}_{3}-\mathrm{H}_{2} \mathrm{O}$ "without $\mathrm{H}_{2} \mathrm{~S}$ " have been irradiated with a $10 \mathrm{keV}$ electron beam and subsequently warmed to room temperature. First results show that the addition of sulfur strongly affects the slope in the Vis-NIR spectral range (M. J. Poston et al. 2017, in preparation). We have previously reported that adding $\mathrm{H}_{2} \mathrm{~S}$ leads to a significant change in the chemistry induced by electron irradiation (Mahjoub et al. 2016). Multiple sulfurous radiation products were detected, but all of them are transparent in the visible-NIR spectral range and cannot explain the observed reddening caused by the presence of sulfur in the initial ice samples. Here, we present new results supporting the formation of sulfur allotropes in the "with $\mathrm{H}_{2} \mathrm{~S}$ " samples. Contrasting the Temperature Programmed Desorption (TPD) curves of samples "with $\mathrm{H}_{2} \mathrm{~S}$ " against samples "without $\mathrm{H}_{2} \mathrm{~S}$ " permits the detection of $\mathrm{S}_{2}, \mathrm{~S}_{3}, \mathrm{~S}_{4}$, and $\mathrm{CH}_{3}-\mathrm{S}-\mathrm{CH}_{3}$, $\mathrm{CH}_{3}-\mathrm{S}_{2}-\mathrm{CH}_{3}, \mathrm{CH}_{3}-\mathrm{SO}_{2}-\mathrm{CH}_{3}$. The implication of this sulfur chemistry on the colors of Jupiter Trojans and KBOs, and the bimodality in spectral slopes of these two groups, is discussed.

This paper is structured as follows. In Section 2 we describe the experimental procedure. Our results are presented in Section 3. The astrophysical implications of this research are discussed in Section 4.

\section{Experimental Methodology}

Electron irradiation experiments were carried out using the Icy Worlds Simulation Laboratory at the Jet Propulsion Laboratory. A detailed description of the facilities and the capabilities of this laboratory can be found in Hand \& Carlson (2011). The experimental setup consisted of a high-vacuum stainless steel chamber pumped by a Varian Turbo and backed by oil-free pumps (pressure after overnight pumping about $1 \times 10^{-8}$ torr). The ices were vapor-deposited on a substrate attached to the cold finger of a closed-cycle helium cryostat (ARS model DE-204). An external manifold was used to prepare gas mixtures prior to deposition. The ice film was grown by leaking the gas mixture into the chamber and forming ices on the substrate, which was held at $50 \mathrm{~K}$. Most of the gas deposited directly, but a small fraction did not, resulting in a rise of chamber pressure of a few $10^{-8}$ torr. High-energy electrons $(10 \mathrm{keV})$ were directed at the ice with a typical beam current of $0.5 \mu \mathrm{A}$. All studied ices were submitted to the same fluence of electron energy $\sim 2 \times 10^{21} \mathrm{eV} \mathrm{cm}^{-2}$. Radiation fluences were scaled to the outer-solar system based on the electron flux at $1 \mathrm{au}$, which was deduced from values given in Bennett et al. (2013). We found that the total fluence received by our ice samples corresponds to a timescale of $0.2 \mathrm{Myr}$ for an object at $5 \mathrm{au}$ and $1.8 \mathrm{Myr}$ at $15 \mathrm{au}$. In the present work we focus our discussion on electron and thermal processing of $\mathrm{H}_{2} \mathrm{~S}-\mathrm{CH}_{3} \mathrm{OH}-\mathrm{NH}_{3}-\mathrm{H}_{2} \mathrm{O} \quad(3: 3: 3: 1) \quad$ "with $\mathrm{H}_{2} \mathrm{~S}$ " and $\mathrm{CH}_{3} \mathrm{OH}-\mathrm{NH}_{3}-\mathrm{H}_{2} \mathrm{O}$ (3:3:1) "without $\mathrm{H}_{2} \mathrm{~S}$ " mixed ices, which are expected for ices in KBOs and Trojans. As discussed in Mahjoub et al. (2016), the chemical composition of ice films calculated using infrared spectra and bond strengths of deposited molecules is different from the gas phase mixture. The compositions of the ices as estimated from the respective column densities are: $\mathrm{CH}_{3} \mathrm{OH}-\mathrm{NH}_{3}-\mathrm{H}_{2} \mathrm{O}=73: 12: 15$ for the "without $\mathrm{H}_{2} \mathrm{~S}$ " ice and $\mathrm{H}_{2} \mathrm{~S}-\mathrm{CH}_{3} \mathrm{OH}-\mathrm{NH}_{3}-\mathrm{H}_{2} \mathrm{O}=7: 35: 17: 41$ for the "with $\mathrm{H}_{2} \mathrm{~S}$ " sample. After irradiation for $19 \mathrm{hr}$ at $50 \mathrm{~K}$, samples were warmed to $120 \mathrm{~K}$ at $0.5 \mathrm{~K}$ minute $^{-1}$ and held there for 1 additional hour under continued electron irradiation. After the electron irradiation was concluded, the samples were warmed at $0.5 \mathrm{~K} \mathrm{minute}^{-1}$ to $300 \mathrm{~K}$. This experimental procedure was chosen to simulate the irradiation and heating history of an icy surface scattered from the primordial Kuiper Belt region to the Jupiter Trojans region. The chemical evolution of the ice was monitored with a Midac Fourier transform infrared spectrometer covering a wavenumber range $400-7000 \mathrm{~cm}^{-1}$ at $2 \mathrm{~cm}^{-1}$ resolution. with 1024 scans accumulated. The angle of incidence of the infrared beam was $\sim 16^{\circ}$ to the normal of the ice film. The ice film was not optically thick: infrared light passed through the ice film and was reflected back through the film by the gold mirror to the detector, where the intensity measured was divided by the intensity of reflected light from the gold mirror alone, the spectrum of which was collected just prior to the ice deposition process. The thickness of the ice films has been estimated to between 1 and $2 \mu \mathrm{m}$ (Mahjoub et al. 2016).

The TPD technique is widely used to study chemistry in irradiated ices for application to interstellar medium and solar system icy bodies. A Stanford Research Systems RGA 200 quadrupole mass spectrometer was used to monitor the gaseous species released when the irradiated ices were warmed. The mass spectrometer operated with a $70 \mathrm{eV}$ electron impact ionization method scanning $\mathrm{m} / \mathrm{z}, 1-200$. TPD spectra correspond to the measurement of masses of molecular species released over the temperature range 50-300 K. The comparison between species desorbed from the samples "with $\mathrm{H}_{2} \mathrm{~S}$ " and "without $\mathrm{H}_{2} \mathrm{~S}$ " helps the assignment of masses observed in RGA mass spectra to specific molecular species. 

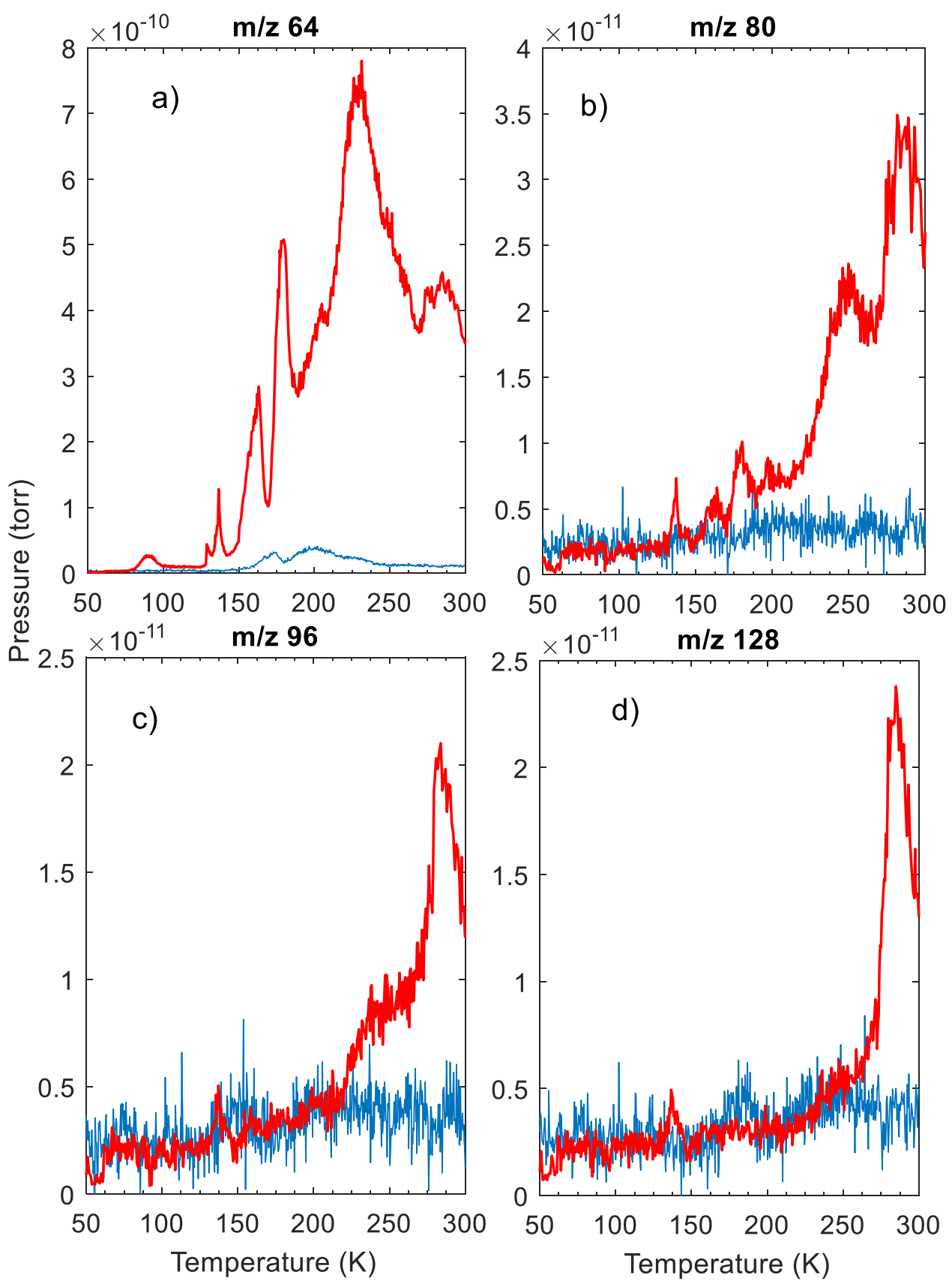

Figure 1. TPD curves corresponding to the irradiated "with $\mathrm{H}_{2} \mathrm{~S}$ " (red curves) and "without $\mathrm{H}_{2} \mathrm{~S}$ " (blue curves) experiments. Essentially no features are seen in the "without $\mathrm{H}_{2} \mathrm{~S}$ " TPD data. The ion currents at $m / z, 64,96$, and 128 correspond, respectively, to $\mathrm{S}_{2}+\mathrm{SO}_{2}, \mathrm{~S}_{3}$, and $\mathrm{S}_{4}$. A possible assignment for mass 80 is $\mathrm{S}_{2} \mathrm{O}+\mathrm{SO}_{3}$.

\section{Results}

All the sulfur-bearing molecules detected via infrared spectroscopy by Mahjoub et al. (2016) are confirmed by TPD measurements: $\mathrm{OCS}, \mathrm{SO}, \mathrm{SO}_{2}, \mathrm{CS}, \mathrm{CS}_{2}$. For brevity, we present here only newly detected molecules. Figure 1 shows TPD curves of irradiated ice mixtures "with $\mathrm{H}_{2} \mathrm{~S}$ " and "without $\mathrm{H}_{2} \mathrm{~S}$ " recorded for $\mathrm{m} / \mathrm{z} 64,80,96$, and 128 . For all these masses, a significant signal is detected only in the samples "with $\mathrm{H}_{2} \mathrm{~S}$," which constrains the assignment of these masses to molecules containing at least one $\mathrm{S}$ atom. The mass $\mathrm{m} / \mathrm{z} 64$ can be assigned to either $\mathrm{SO}_{2}$ or $\mathrm{S}_{2}$. $\mathrm{SO}_{2}$ has been detected by midIR spectroscopy in the samples "with $\mathrm{H}_{2} \mathrm{~S}$ " (Mahjoub et al. 2016).
To find out if $\mathrm{SO}_{2}$ alone is responsible for the signal at $m / z$ 64, we compare the TPD spectra of $\mathrm{m} / \mathrm{z} 64$ and $\mathrm{m} / \mathrm{z} 48$, corresponding to $\mathrm{SO}$, the principle fragment of $\mathrm{SO}_{2}$ (Figure 2). Figure 2 also presents a comparison between TPD curves at $\mathrm{m} / \mathrm{z} 48$ for the "with $\mathrm{H}_{2} \mathrm{~S}$ " and "without $\mathrm{H}_{2} \mathrm{~S}$ " samples. A significant signal is observed only from the "with $\mathrm{H}_{2} \mathrm{~S}$ " sample, indicating that this mass is mostly likely due to sulfur-containing molecules.

Mass 48 corresponds to the sulfur monoxide molecule, SO, which could be directly desorbed from the irradiated sample, or could be a product of fragmentation of $\mathrm{SO}_{2}, \mathrm{~S}_{2} \mathrm{O}$, and $/$ or $\mathrm{SO}_{3}$. $\mathrm{CH}_{3} \mathrm{SH}$ could also be produced in our samples and would contribute to the signal at $\mathrm{m} / \mathrm{z} 48$. Deconvolution of contributions from all these possible candidates to mass 48 is not possible with these data. However, since $S_{2}$ does not produce 


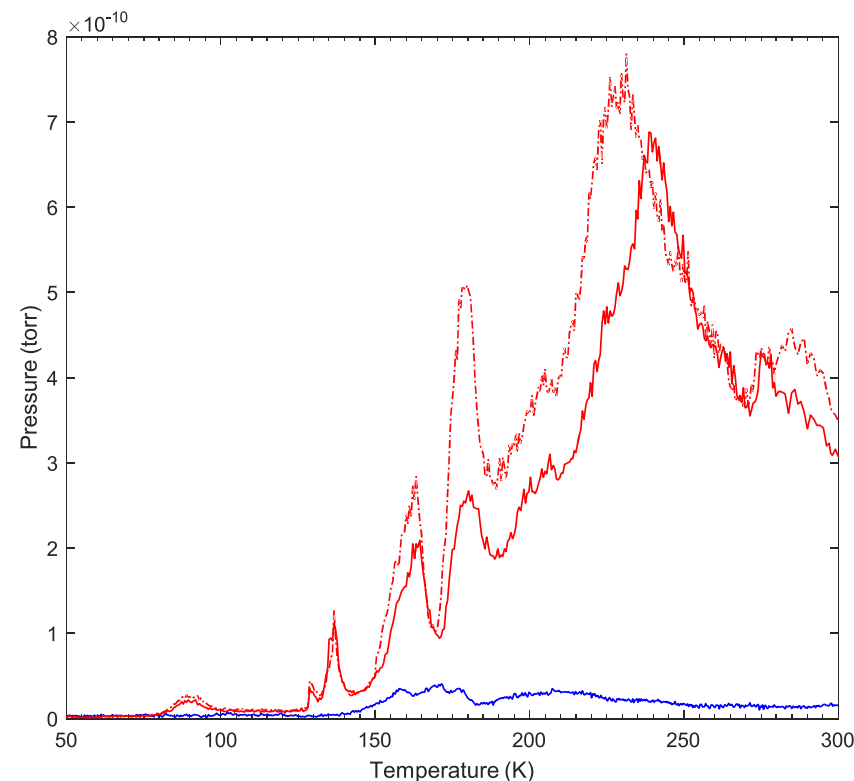

Figure 2. TPD curves at $m / z 48$ corresponding to the irradiated "with $\mathrm{H}_{2} \mathrm{~S}$ " (red solid curve) and "without $\mathrm{H}_{2} \mathrm{~S}$ " (blue curve) experiments and at $m / z, 64$ (dashed red curve) for the "with $\mathrm{H}_{2} \mathrm{~S}$ " experiment.

fragments at mass 48, comparison between mass 64 and 48 (Figure 2) provides information on the likelihood that $S_{2}$ is present. At some temperatures the two peaks move synchronously, but at $230-240 \mathrm{~K}$ the two signals reach maxima with a separation of $9 \mathrm{~K}$. This shift indicates that the two masses are decoupled at this temperature, which indicates that the desorption at $T=230 \mathrm{~K}$ could be due to other molecules in addition to $\mathrm{SO}_{2}$. While these observations are circumstantial evidence of the possible formation of $S_{2}$ in the irradiated "with $\mathrm{H}_{2} \mathrm{~S}$ " sample, they are not enough for an actual detection.

Figure 3 compares TPD curves at $\mathrm{m} / \mathrm{z}, 64$ and $\mathrm{m} / \mathrm{z} \quad 68$, corresponding to ${ }^{34} \mathrm{~S}_{2}$ isotopomer of $\mathrm{S}_{2}$. It is unlikely that mass 68 corresponds to $\mathrm{S}^{18} \mathrm{O}_{2}$ since the natural abundance of ${ }^{18} \mathrm{O}$ is very low $(0.2 \%)$ compared to ${ }^{34} \mathrm{~S}(4.29 \%)$. The TPD curve at $\mathrm{m} / \mathrm{z} 68$ does not contain a peak at $178 \mathrm{~K}$, indicating that this peak is probably not due to $\mathrm{S}_{2}$. The broad peak at $230 \mathrm{~K}$ is present in both TPD curves of $m / z 68$ and 64. The feature at $230 \mathrm{~K}$ may be primarily due to $S_{2}$ desorption. Considering all temperatures, we conclude that the signal at $\mathrm{m} / \mathrm{z}, 64$ corresponds to at least $\mathrm{S}_{2}$ and $\mathrm{SO}_{2}$ contributions.

$\mathrm{S}_{2}$ was reported as a UV photochemical product of $\mathrm{H}_{2} \mathrm{~S}-\mathrm{H}_{2} \mathrm{O}$ ice mixture (Jimenez-Escobar \& Caro 2011) and $\mathrm{X}$-ray irradiation of pure $\mathrm{H}_{2} \mathrm{~S}$ with a relatively high ratio $\left[\mathrm{S}_{2}\right] /\left[\mathrm{H}_{2} \mathrm{~S}\right]$ equaling 0.16 (Jimenez-Escobar et al. 2012). $\mathrm{S}_{2}$ could be formed by several mechanisms in our irradiation experiment "with $\mathrm{H}_{2} \mathrm{~S}$." $\mathrm{S}_{2}$ could be a product of a reaction between two HS radicals (which are products of irradiation of $\mathrm{H}_{2} \mathrm{~S}$ ), via the reaction $\mathrm{HS}+\mathrm{HS} \rightarrow \mathrm{H}_{2}+\mathrm{S}_{2}$, or could form in a reaction between atomic sulfur atoms via the reaction $\mathrm{S}+\mathrm{S} \rightarrow \mathrm{S}_{2}$. In addition, OCS formed by irradiation could subsequently react with a sulfur atom and produce the sulfur dimer: $\mathrm{S}+\mathrm{OCS} \rightarrow \mathrm{S}_{2}+\mathrm{CO}$. Finally, $\mathrm{S}_{2}$ could also be produced by dissociation of $\mathrm{H}_{2} \mathrm{~S}_{2}$. While $\mathrm{H}_{2} \mathrm{~S}_{2}$ was not originally reported in Mahjoub et al. (2016), we will report the possible detection of this molecule in our samples by IR spectroscopy later in this section.

In Figure 1(b) the comparison between TPD curves of "with $\mathrm{H}_{2} \mathrm{~S}$ " and "without $\mathrm{H}_{2} \mathrm{~S}$ " samples at $\mathrm{m} / \mathrm{z} 80$ indicates a sulfur-

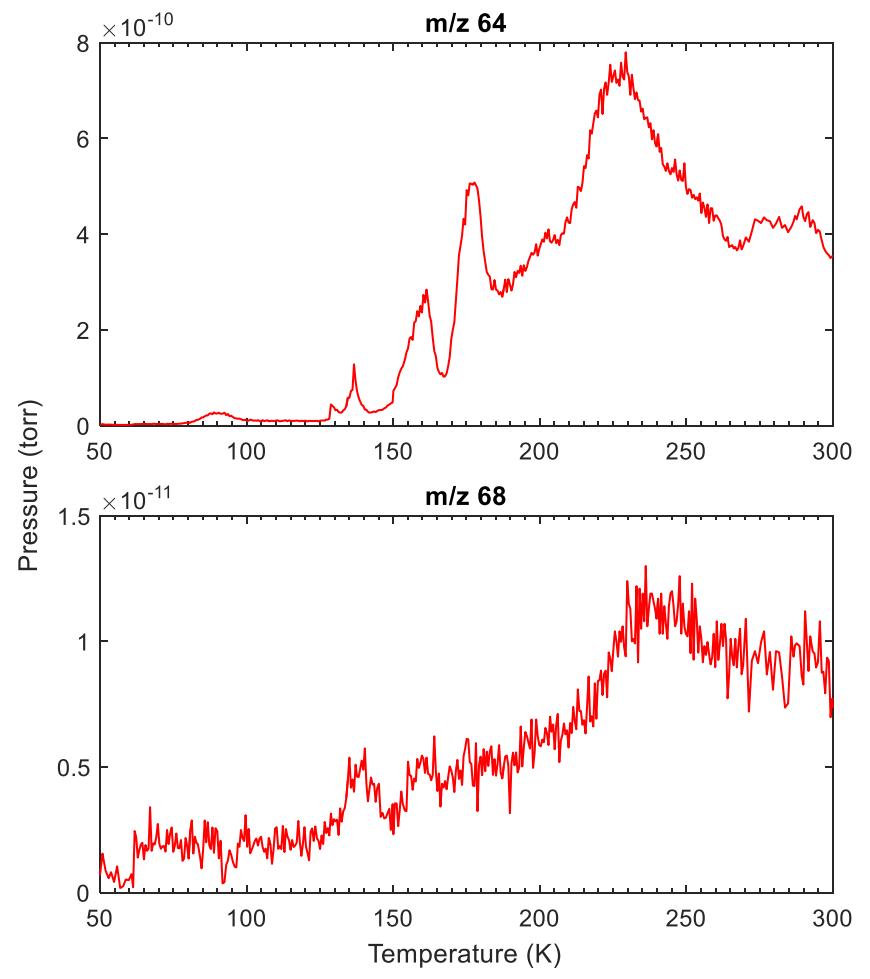

Figure 3. Comparison between TPD spectra at $m / z, 64$ and $m / z 68$.

containing molecule. The sulfurous compounds that could be responsible for mass 80 are $\mathrm{S}_{2} \mathrm{O}$ and $\mathrm{SO}_{3}$. The principal fragment from both $\mathrm{SO}_{3}$ and $\mathrm{S}_{2} \mathrm{O}$ is $\mathrm{SO}(\mathrm{m} / z, 48)$. As discussed above, the signal at $m / z, 48$ could derive from $\mathrm{SO}$ released from the ice via fragmentation of $\mathrm{SO}_{2}, \mathrm{SO}_{3}$, and $\mathrm{S}_{2} \mathrm{O}$, as well as $\mathrm{CH}_{3} \mathrm{SH}$. This hinders the identification of $\mathrm{SO}_{3}$ and $\mathrm{S}_{2} \mathrm{O}$ using the fragmentation pattern. Moreover, the signal at mass 84 $\left({ }^{34} \mathrm{~S}_{2} \mathrm{O}\right)$ is very weak and does not allow confirmation of the formation of $\mathrm{S}_{2} \mathrm{O}$. Irradiation experiments using isotopes labeled $\mathrm{H}_{2} \mathrm{~S}$ are needed to infer whether both $\mathrm{SO}_{3}$ and $\mathrm{S}_{2} \mathrm{O}$ contribute to the signal at mass 80 . Both $\mathrm{SO}_{3}$ and $\mathrm{S}_{2} \mathrm{O}$ have been produced in electric discharge applied to $\mathrm{SO}_{2}$ (Hopkins et al. 1973). $\mathrm{SO}_{3}$ was also observed as a product of ion bombardment of $\mathrm{SO}_{2}$ pure ice (Gomis \& Strazzulla 2008).

Figures 1(c) and (d) of $\mathrm{m} / z, 96$ and 128 both peak in desorption around $T=282 \mathrm{~K}$. We assign these two masses to the allotropes $S_{3}$ and $S_{4}$, respectively. $S_{3}$ is detected in irradiated $\mathrm{H}_{2} \mathrm{~S}$ (Jimenez-Escobar \& Caro 2011). The desorption behavior observed at $\mathrm{m} / \mathrm{z}, 96$ is similar to $\mathrm{S}_{3}$ after UV irradiation of pure $\mathrm{H}_{2} \mathrm{~S}$ (Jimenez-Escobar \& Caro 2011), with a single peak between 250 and $300 \mathrm{~K}$. Tetrasulfur, $\mathrm{S}_{4}$, can be formed during co-deposition of $S_{2}+K r$, forming a red film. Films of $S_{2}$ in $\mathrm{Kr}$ also form $\mathrm{S}_{4}$ when irradiated with visible light (Meyer \& Stroyerh 1972). Hassanzadeh \& Andrews (1992) ruled out the formation mechanism of $\mathrm{S}_{4}$ by recombination of two $\mathrm{S}_{2}$ molecules in gas phase, but this reaction is possible in a matrix surface (Hopkins et al. 1973), which explains the formation of $\mathrm{S}_{4}$ in co-deposited $\mathrm{S}_{2}-\mathrm{Kr}$ mixture. Another reaction pathway could be: $\mathrm{S}+\mathrm{S}_{3} \rightarrow \mathrm{S}_{4} . \mathrm{S}_{3}$ and $\mathrm{S}_{4}$ have been detected as a product of electric discharge in gaseous $\mathrm{SO}_{2}$ (Hopkins et al. 1973).

Sulfur allotropes $S_{2}, S_{3}$, and $S_{4}$ have very weak infrared absorption bands at wavenumbers between 200 and $600 \mathrm{~cm}^{-1}$. This makes their detection in our samples by IR spectroscopy very difficult at low column densities. 

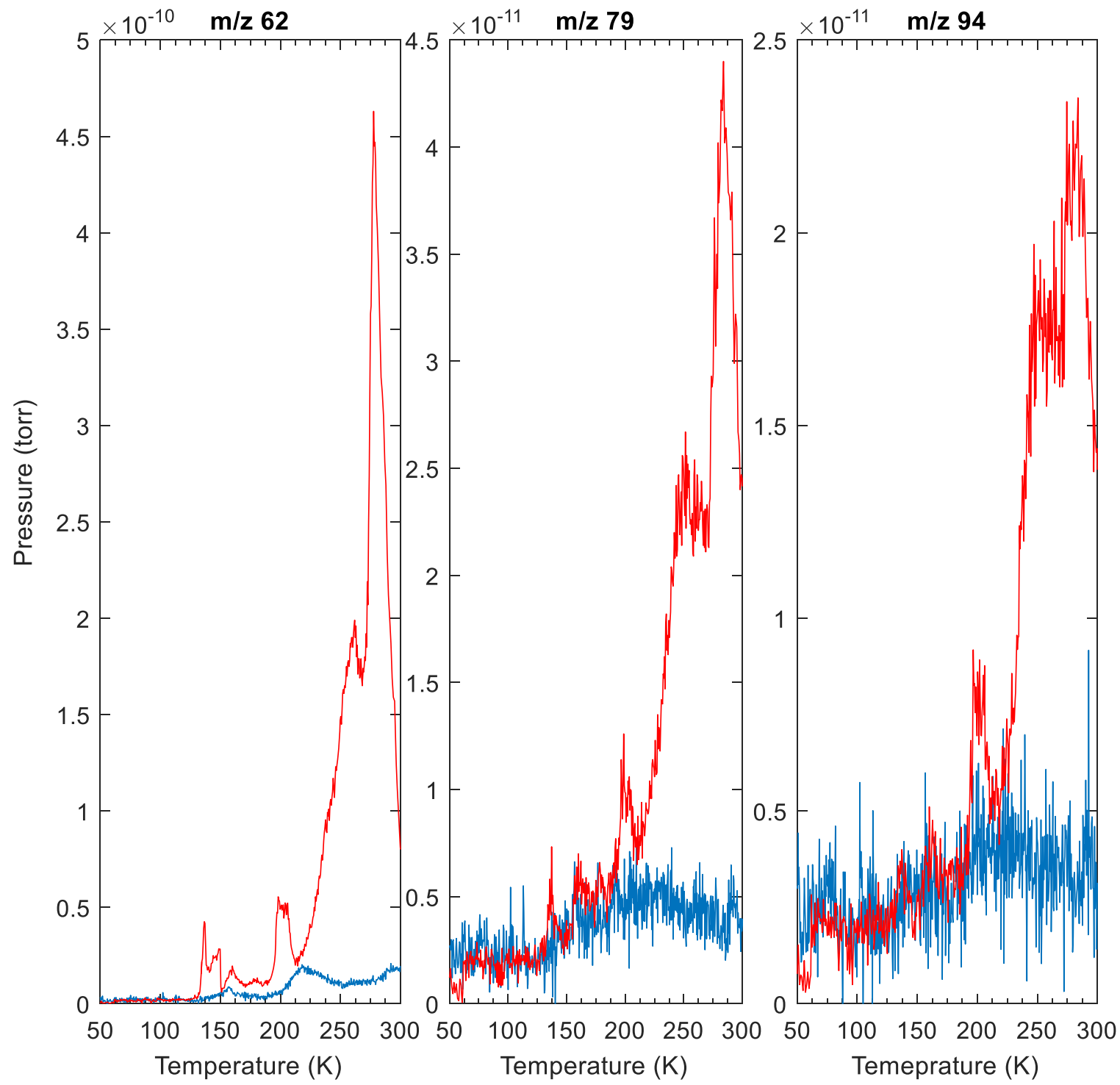

Figure 4. TPD curves corresponding to the irradiated "with $\mathrm{H}_{2} \mathrm{~S}$ " (red curves) and "without $\mathrm{H}_{2} \mathrm{~S}$ " (blue curves) experiments. Ion currents at $m / z 62$ and 94 correspond, respectively, to $\mathrm{CH}_{3} \mathrm{SCH}_{3}$ and $\mathrm{CH}_{3} \mathrm{~S}_{2} \mathrm{CH}_{3}+\mathrm{CH}_{3} \mathrm{SO}_{2} \mathrm{CH}_{3} ; \mathrm{m} / 2.79$ assigned their principal fragments to $\mathrm{CH}_{3} \mathrm{~S}_{2}+\mathrm{CH}_{3} \mathrm{SO}_{2}$.

It is noteworthy that we did not detect any chains containing more than four $\mathrm{S}$ atoms in our TPD spectra, in spite of scanning to $\mathrm{m} / \mathrm{z} 200\left(\mathrm{~S}_{5}\right.$ and $\mathrm{S}_{6}$ have molar masses of 160 and 192 , respectively, while those of $S_{7}$ and $S_{8}$ are 224 and 256, which are beyond our range). The non-detection in the RGA mass spectrometer of sulfur allotropes higher than $\mathrm{S}_{4}$ indicates that the signal corresponding to $S_{2}, S_{3}$, and $S_{4}$ could not be due to fragments of higher sulfur chains. This absence of sulfur chains in the mass spectra does not rule out the formation of $S_{x}$ polymers $(x>4)$. These larger polymers like $S_{8}$ are less volatile, and if they were produced they likely remained in the organic residue left on the substrate at room temperature. A future study of the residue may clarify whether larger sulfur polymers do form by irradiation.

In addition to short sulfur chains we detected other organosulfur molecules (i.e., $\mathrm{C}_{x} \mathrm{H}_{y} \mathrm{~S}_{z}$ ). Figure 4 shows peaks at $m / z 62$ and 94 that provide evidence for the production of other sulfurcontaining compounds. Mass 62 could be assigned to $\mathrm{CH}_{3} \mathrm{SCH}_{3}$. This assignment is supported by the detection of fragment $\mathrm{CH}_{3} \mathrm{~S}(\mathrm{~m} / 2,47)$ of $\mathrm{C}_{2} \mathrm{H}_{6} \mathrm{~S}$ at $T \sim 290 \mathrm{~K}$. The ratio of signal $62 / 47$ at this temperature is 1.17 , close to the ratio of signal $62 / 47=1.05$ by electron impact mass spectrometry of $\mathrm{CH}_{3} \mathrm{SCH}_{3}$ (NIST Mass Spectra Data Center \& Stein 2008). We cannot totally exclude the contribution of $\mathrm{H}_{2} \mathrm{COS}$ (sulfine) to the signal detected at $m / z$ 64, though sulfine is unstable and has a very short lifetime (Arnaud et al. 1999). Mass 94 could be assigned to $\mathrm{CH}_{3}-\mathrm{SO}_{2}-\mathrm{CH}_{3}$ (dimethyl sulfone) and/or $\mathrm{CH}_{3}-\mathrm{S}_{2}-\mathrm{CH}_{3}$ (dimethyl disulfide). Both molecules have a fragment at mass 79 (NIST Mass Spectra Data Center \& Stein 2008). We have detected a signal at $\mathrm{m} / z .79$ exclusively in the "with $\mathrm{H}_{2} \mathrm{~S}$ " sample (Figure 4). A close comparison between TPD spectra at 79 and 94 shows a ratio $79 / 94 \sim 1.4$ at $T=255 \mathrm{~K}$ and $\sim 1.9$ at $284 \mathrm{~K}$. The $79 / 94$ ratios in NIST mass spectra of $\mathrm{CH}_{3} \mathrm{SO}_{2} \mathrm{CH}_{3}$ and $\mathrm{CH}_{3}-\mathrm{S}_{2}-\mathrm{CH}_{3}$ are, respectively, $\sim 2$ and 0.57 . The ratio $79 / 94$ of $\mathrm{CH}_{3} \mathrm{SO}_{2} \mathrm{CH}_{3}$ is fairly in agreement with the peak at $284 \mathrm{~K}$, consistent with this desorption peak being dominated by dimethyl sulfone. The peak at $255 \mathrm{~K}$ could not be explained by $\mathrm{CH}_{3} \mathrm{SO}_{2} \mathrm{CH}_{3}$ alone, so we tentatively assign this peak to the desorption of both $\mathrm{CH}_{3}-\mathrm{S}_{2}-\mathrm{CH}_{3}$ and $\mathrm{CH}_{3} \mathrm{SO}_{2} \mathrm{CH}_{3}$. The detection of large organosulfur molecules indicates that a complex carbon-sulfur chemistry is driven by irradiation of simple methanolhydrogen-sulfide-containing ices. $\mathrm{H}_{2} \mathrm{~S}$ may have affected the 
Table 1

Summary of Sulfurous Species Detected by Comparing TPD Measurements of Samples "with $\mathrm{H}_{2} \mathrm{~S}$ " with Samples "Without $\mathrm{H}_{2} \mathrm{~S}$ "

\begin{tabular}{lll}
\hline \hline Mass & Assignment & $T(\mathrm{~K})$ of Principal Desorption Peaks \\
\hline 62 & $\mathrm{CH}_{3} \mathrm{SCH}_{3}, \mathrm{H}_{2} \mathrm{CSO} ?$ & 260,278 \\
64 & $\mathrm{~S}_{2}, \mathrm{SO}_{2}$ & $163,179,230$ \\
80 & $\mathrm{~S}_{2} \mathrm{O} ?, \mathrm{SO}_{3} ?$ & 245,290 \\
94 & $\mathrm{CH}_{3} \mathrm{SO}_{2} \mathrm{CH}_{3}, \mathrm{CH}_{3} \mathrm{~S}_{2} \mathrm{CH}_{3}$ & 255,284 \\
96 & $\mathrm{~S}_{3}$ & 282 \\
128 & $\mathrm{~S}_{4}$ & 282 \\
\hline
\end{tabular}

Note. The question mark stands for possible assignment.

carbon chemistry in the sample by involving radicals and ions formed by irradiation of $\mathrm{CH}_{3} \mathrm{OH}$ in sulfur-related chemical reactions. In addition to the possible effect of this chemistry on the colors of the irradiation residue, this chemistry could be astrobiologically relevant because of its role in the generation of diverse organic species. Detected species are summarized in Table 1.

Figure 5 shows IR spectra of samples "with $\mathrm{H}_{2} \mathrm{~S}$ " before and after irradiation. After two hours of irradiation, a band appears at $2507 \mathrm{~cm}^{-1}$. One possible assignment of this band is the $\mathrm{H}_{2} \mathrm{~S}_{2}$ dimer or $\mathrm{H}_{2} \mathrm{~S}_{2}-\mathrm{H}_{2} \mathrm{~S}$ complex, based on previous assignments by Isoniemi et al. (1999). The $\mathrm{S}-\mathrm{H}$ stretching mode of $\mathrm{H}_{2} \mathrm{~S}_{2}$ is in the same position as the $\mathrm{H}_{2} \mathrm{~S}$ band (in the mixture $\mathrm{H}_{2} \mathrm{~S}-\mathrm{H}_{2} \mathrm{O}$ ) at $2550 \mathrm{~cm}^{-1}$, precluding direct detection of $\mathrm{H}_{2} \mathrm{~S}_{2}$. This assignment needs to be definitively confirmed by isotopic effect studies. $\mathrm{H}_{2} \mathrm{~S}_{2}$ could be produced by a reaction between two HS radicals. The maximum of the $2507 \mathrm{~cm}^{-1}$ band appears after two hours of irradiation, after which further irradiation decreases this band. At the end of the experiment (after $19 \mathrm{hr}$ of irradiation) this band disappears completely. $\mathrm{H}_{2} \mathrm{~S}_{2}$ formed early in the irradiation is likely dissociated by the irradiation and may react to form larger molecules. Isoniemi et al. (1999) studied UV photolysis of $\mathrm{H}_{2} \mathrm{~S}_{2}$ ice in an Ar matrix and concluded that this photochemistry leads to the formation of the $\mathrm{HS}_{2}$ radical and $\mathrm{S}_{2}$. Dissociation of $\mathrm{H}_{2} \mathrm{~S}_{2}$ presumably contributes to the production of $\mathrm{S}_{2}$ detected by TPD. The infrared band strength of $\mathrm{H}_{2} \mathrm{~S}_{2}$ is not reported in the literature, which prohibits the determination of the column density of $\mathrm{H}_{2} \mathrm{~S}_{2}$ produced in our experiment.

\section{Discussion}

\subsection{Applications to Jupiter Trojans and KBOs}

This work aims to test the hypothesis correlating the color bimodality in Jupiter Trojans and small KBOs with the sulfur chemistry in the early solar system (Wong \& Brown 2016). $\mathrm{S}_{x}$ chains have been considered as possible candidate sources of the coloring on the surface of Io (Nelson \& Hapke 1978; Spencer et al. 1997), as well as the Great Red Spot of Jupiter. Organic macromolecules produced by irradiation of carbonand nitrogen-containing ices are also proposed to explain the red slopes observed on the surfaces of small icy bodies. Sulfur small chains have the particularity of changing colors when submitted to thermal or irradiation processing. As explained in the review of sulfur color properties in the introduction, this color change is a function of the size of the sulfur polymer. This particularity could explain the difference between the colors of Trojans and KBOs. It is important to clarify that we are not claiming here that sulfur chains are alone responsible

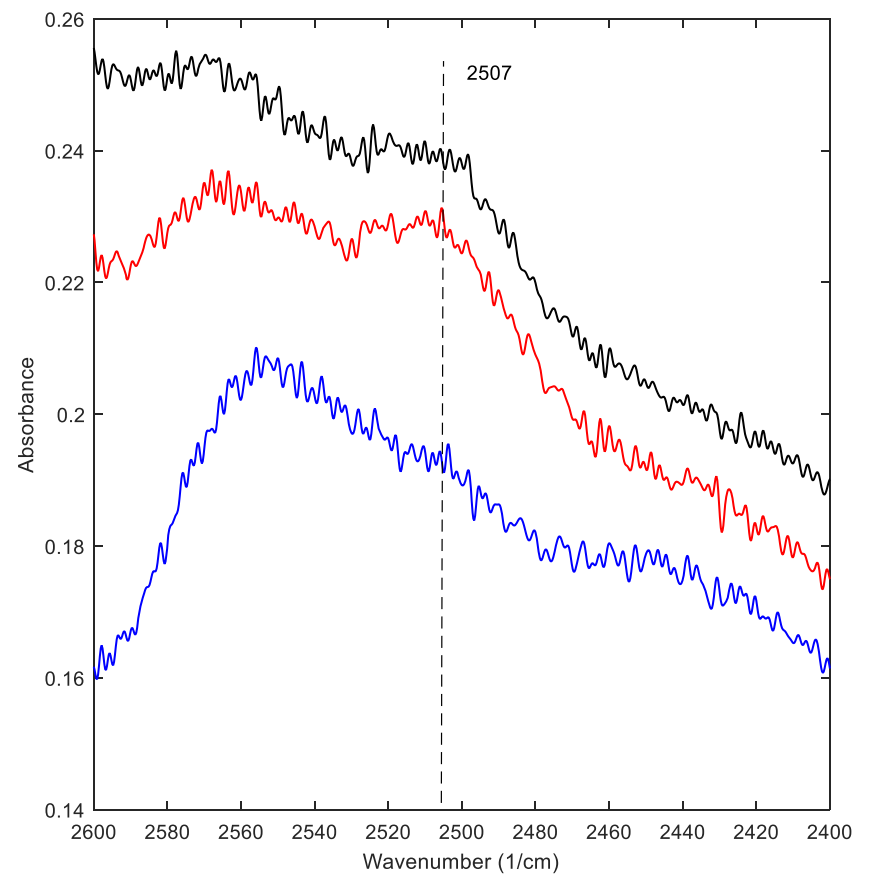

Figure 5. Infrared spectrum around $2550 \mathrm{~cm}^{-1}$ of the sample "with $\mathrm{H}_{2} \mathrm{~S}$ " before (blue), after $2 \mathrm{hr}$ (red), and after $5 \mathrm{hr}$ (black) of electron irradiation. The band at $2507 \mathrm{~cm}^{-1}$ is assigned to an $\mathrm{H}_{2} \mathrm{~S}_{2}$ dimer. This band appears after $2 \mathrm{hr}$ of electron irradiation (red) and decreases significantly after $5 \mathrm{hr}$ (black).

for the spectral colors of Trojans and KBOs. An accurate estimation of the production rates of these species by irradiation and measurements of their quantitative optical properties (refractive indices) is needed to quantitatively estimate their role as coloring agents.

In the framework of the hypothesis that sulfur irradiation chemistry plays a key role in the color bimodality observed in KBOs and Trojans, our experimental work supports some aspects of the hypothesis:

1. Our laboratory simulations show that sulfur allotropes are produced as a result of electron irradiation of $\mathrm{H}_{2} \mathrm{~S}$-containing mixed ices. It is conceivable that these sulfur materials bring about the red slope observed in Trojans and KBOs.

2. Stored at very low temperatures $(\sim 30,50 \mathrm{~K})$ and in a relatively low irradiation environment, KBOs are expected to preserve unstable small sulfur allotropes. Jupiter Trojans, on the other hand, are exposed to higher irradiation and a warmer environment. The thermal and irradiation processing experienced by Trojans in their migration pathway (as predicted by Nice model) can turn small red allotrope sulfur chains into neutral $\mathrm{S}_{8}$. This is consistent with KBOs being redder than Trojans.

On the other hand, our results rebut one important prediction of that hypothesis:

1. VR KBOs are known to be much brighter than R KBOs and Trojans. Yellow sulfur polymers have a higher albedo compared to samples rich in small sulfur allotropes (like liquid sulfur). Therefore, the transformation of sulfur allotropes into large sulfur polymers could not explain the lower albedo of Trojans.

Detection of these molecules with remote sensing methods from telescopes and spacecraft would be challenging, but is not 
unfeasible. Sulfur allotropes have very weak absorption bands in the far- and mid-IR regions, so they cannot be directly detected by remote infrared spectroscopy. However, these species have intense bands in the ultraviolet and visible ranges: $\mathrm{S}_{2}$ absorbs from the ultraviolet to $500 \mathrm{~nm}$ or greater, with the band center occurring at $360 \mathrm{~nm}$ (Meyer \& Stroyerh 1972; Steudel \& Eckert 2003); $S_{3}$ has a broad absorption band centered at about $400 \mathrm{~nm}$ and extending to $\sim 500 \mathrm{~nm}$; and $\mathrm{S}_{4}$ has a band centered at about $530 \mathrm{~nm}$, an absorption between $460-590 \mathrm{~nm}$ that is attributed to the $\mathrm{C}_{2 v}$ isomer, and a weaker band at $625 \mathrm{~nm}$ (Meyer \& Stroyerh 1972). The molar extinction coefficient of $S_{3}$ is $\sim 10^{-16} \mathrm{~cm}^{2}$ at $400 \mathrm{~nm}$ and is one order of magnitude higher than of $\mathrm{S}_{4}$. Detection of these species by UV and Vis spectroscopy might be possible if they are present with measurable quantities. UV measurements of Trojans and KBOs are rare. (Stern et al. 2014) reported the only UV measurements of KBOs other than Pluto using the Hubble Space Telescope. They reported a UV absorption feature in the spectrum of KBO Haumea centered near $305 \mathrm{~nm}$. They proposed $\mathrm{SO}_{2}$ and $\mathrm{OH}$ as possible candidates for this band. Similar observations in the UV and high-quality spectra in the visible of other KBOs are needed to better constrain the presence of sulfur allotropes on the surface of these objects.

\subsection{Sulfur Irradiation Products in Cometary Materials}

With the exception of $\mathrm{C}_{2} \mathrm{H}_{6} \mathrm{~S}_{2}$ and $\mathrm{C}_{2} \mathrm{H}_{6} \mathrm{SO}_{2}$, the list of sulfurous molecules detected in our experiment either by TPD in this paper or by FTIR spectroscopy (Mahjoub et al. 2016) matches exactly the list of sulfur molecules detected by the ROSINA/DFMS mass spectrometer at 67P (Calmonte et al. 2016). Disulfur, $S_{2}$, is ubiquitous in comets. This molecule was first detected in the UV spectra of comet C/1983 H1 (Ahearn et al. 1983). Since then, $S_{2}$ has been identified in many comets (Swamy \& Wallis 1987; Laffont et al. 1998; Kim et al. 2003). Recently the ROSINA mass spectrometer on board the Rosetta spacecraft detected $\mathrm{S}_{2}$ at a distance of 3.15 au from the Sun, with an abundance of $\sim 10^{-5}$ compared to water (Le Roy et al. 2015). Ahearn et al. (1983) suggested that $S_{2}$ comes from (or is formed very close to) the comet nucleus rather than by gas phase chemistry in the cometary coma. Indeed, $S_{2}$ is observed at distances larger than $3 \mathrm{au}$, where the gas density is very low and collisions are rare, these conditions are not convenient for gas phase recombination reactions. $\mathrm{S}_{3}$ and $\mathrm{S}_{4}$ have also been recently detected in the coma of comet $67 \mathrm{P}$ by the ROSINA/ DFMS mass spectrometer on board the Rosetta spacecraft, and may originate from dust grains (Calmonte et al. 2016). The organo-sulfur molecule $\mathrm{C}_{2} \mathrm{H}_{6} \mathrm{~S}$ has also been detected in the $67 \mathrm{P}$ coma (Calmonte et al. 2016), and the $\mathrm{C}_{2} \mathrm{H}_{6} \mathrm{~S} / \mathrm{H}_{2} \mathrm{~S}$ ratio is estimated to be around $10^{-4}$. Calmonte et al. (2016) discussed the likelihood of radiolysis of $\mathrm{H}_{2} \mathrm{~S}$ in the pre-solar cloud as an origin of sulfurous molecules (particularly $S_{2}$ ). While our experiment does not depict the conditions of the pre-solar nebula, our work shows that radiolysis of ice mixtures containing $\mathrm{CH}_{3} \mathrm{OH}$ and $\mathrm{H}_{2} \mathrm{~S}$ reproduces much of the list of sulfur species detected by Rosetta.

\section{Conclusion}

We present in this article a TPD study of irradiated $\mathrm{CH}_{3} \mathrm{OH}-\mathrm{NH}_{3}-\mathrm{H}_{2} \mathrm{O}$ ("without $\mathrm{H}_{2} \mathrm{~S}$ ") and $\mathrm{H}_{2} \mathrm{~S}-\mathrm{CH}_{3} \mathrm{OH}-\mathrm{NH}_{3}-\mathrm{H}_{2} \mathrm{O}$ ("with $\mathrm{H}_{2} \mathrm{~S}$ ") ices. Small sulfur allotropes $\left(\mathrm{S}_{2}, \mathrm{~S}_{3}, \mathrm{~S}_{4}\right)$ are susceptible to being formed in the mixture "with $\mathrm{H}_{2} \mathrm{~S}$," as well as more complex $\mathrm{H}_{2} \mathrm{~S}_{2}$ and $\mathrm{C}_{x} \mathrm{H}_{y} \mathrm{~S}_{z}$ species. These sulfur polymers could characterize objects formed outside the stability line of $\mathrm{H}_{2} \mathrm{~S}$ and could play a role in the color of KBOs. Produced allotropes become unstable under irradiation and heating conditions, as would be experienced by an object scattered from the primordial KBO reservoir region (beyond $15 \mathrm{au}$ ) to the current Jupiter Trojans location. The small allotropes could convert into larger polymers, principally $S_{8}$, characterized by neutral spectra at wavelengths higher than $500 \mathrm{~nm}$. This may flatten the red slope in the spectral reflectance of these objects. While these results support the chemical credibility of the hypothesis of a $\mathrm{H}_{2} \mathrm{~S}$ role in the color bimodality of Trojans, they are not able to explain some characteristics, such as the high albedo of KBOs compared to Trojans and the lack of absorption bands at visible wavelengths.

The main outcome of this laboratory work is the production and characterization of sulfur compounds that would be fingerprints left after irradiation of an object formed beyond the sublimation line of $\mathrm{H}_{2} \mathrm{~S}$.

This work was conducted at the Jet Propulsion Laboratory, Caltech, under a contract with the National Aeronautics and Space Administration (NASA) and at the Caltech Division of Geological and Planetary Sciences. This work was supported by the Keck Institute of Space Studies (KISS).

We would like to thank the anonymous reviewer for pertinent comments that considerably improved the manuscript.

U.S. Government sponsorship is acknowledged.

\section{ORCID iDs}

Ahmed Mahjoub (iD https://orcid.org/0000-0003-1229-5208

Michael E. Brown (iD https://orcid.org/0000-0002-8255-0545 Bethany L. Ehlmann (iD https://orcid.org/0000-00022745-3240

Kevin P. Hand (iD https://orcid.org/0000-0002-3225-9426

\section{References}

Ahearn, M. F., Feldman, P. D., \& Schleicher, D. G. 1983, ApJL, 274, L99 Arnaud, R., Juvin, P., \& Vallee, Y. 1999, J Org Chem, 64, 8880 Baklouti, D., Schmitt, B., \& Brissaud, O. 2008, Icar, 194, 647 Bennett, C. J., Pirim, C., \& Orlando, T. M. 2013, ChRv, 113, 9086 Brabson, G. D., Mielke, Z., \& Andrews, L. 1991, JPhCh, 95, 79 Brown, M. E., Schaller, E. L., \& Fraser, W. C. 2011, ApJL, 739, 5 Calmonte, U., Altwegg, K., Balsiger, H., et al. 2016, MNRAS, 462, S253 Emery, J. P., Burr, D. M., \& Cruikshank, D. P. 2011, AJ, 141, 18 Fraser, W. C., Brown, M. E., Morbidelli, A., Parker, A., \& Batygin, K. 2014, ApJ, 782, 14

Gomis, O., \& Strazzulla, G. 2008, Icar, 194, 146

Gradie, J., \& Veverka, J. 1980, Natur, 283, 840

Hand, K. P., \& Carlson, R. W. 2011, Icar, 215, 226

Hassanzadeh, P., \& Andrews, L. 1992, JPhCh, 96, 6579

Hopkins, A. G., Tang, S. Y., \& Brown, C. W. 1973, J Am Chem Soc, 95, 3486 Isoniemi, E., Pettersson, M., Khriachtchev, L., Lundell, J., \& Rasanen, M. 1999, JPCA, 103, 679

Jimenez-Escobar, A., \& Caro, G. M. M. 2011, A\&A, 536, 11

Jimenez-Escobar, A., Caro, G. M. M., Ciaravella, A., et al. 2012, ApJL, 751, 5

Kim, S. J., Ah'earn, M. F., Wellnitz, D. D., Meier, R., \& Lee, Y. S. 2003, Icar, 166,157

Laffont, C., Boice, D. C., Moreels, G., et al. 1998, GeoRL, 25, 2749

Le Roy, L., Altwegg, K., Balsiger, H., et al. 2015, A\&A, 583, 12

Mahjoub, A., Poston, M. J., Hand, K. P., et al. 2016, ApJ, 820, 9

Meyer, B. 1976, ChRv, 76, 367

Meyer, B., Oommen, T. V., \& Jensen, D. 1971, JPhCh, 75, 912

Meyer, B., \& Stroyerh, T. 1972, JPhCh, 76, 3968 
Morbidelli, A., Levison, H. F., Tsiganis, K., \& Gomes, R. 2005, Natur, 435, 462

Nelson, R. M., \& Hapke, B. W. 1978, Icar, 36, 304

Nesvorny, D., Vokrouhlicky, D., \& Morbidelli, A. 2013, ApJ, 768, 8

NIST Mass Spec Data Center, \& Stein, S. E. 2008, in NIST Chemical WebBook, Standard NIST reference database Number 69, ed. P. J. Linstrom \& W. G. Mallard (Gaithersburg, MD: National Institute of Standards and Technology), 20899 http://webbook.nist.gov
Quirico, E., Moroz, L. V., Schmitt, B., et al. 2016, Icar, 272, 32

Spencer, J. R., McEwen, A. S., McGrath, M. A., et al. 1997, Icar, 127, 221

Stern, S. A., Cunningham, N. J., \& Schindhelm, E. 2014, AJ, 147, 6

Steudel, R., \& Eckert, B. 2003, in Topics in Current Chemistry, ed. R. Steudel. (Berlin: Springer), 1

Swamy, K. S. K., \& Wallis, M. K. 1987, MNRAS, 228, 305

Wong, I., \& Brown, M. E. 2016, AJ, 152, 8

Wong, I., \& Brown, M. E. 2017, AJ, 153, 9 